was associated with a higher peripheral joint damage score (by mSS, adjusted $\beta=29.5,95 \% \mathrm{Cl} 8.2,50.8, p=0.007$, Table 1 ), while the association between prolonged time spent sitting and lower mSS was of borderline significance $(p=0.085$, Table 1$)$. Additionally, occupations that required higher finger dexterity were associated with higher $\mathrm{mSS}$ (adjusted $\beta=5.4,95 \% \mathrm{Cl} 1.6,9.2, p=0.005$, Table 2). Regarding axial damage, occupations that involved prolonged walking/running were associated with a higher mSASSS score (adjusted $\beta=4.4,95 \% \mathrm{Cl} 0.1,8.7$, $p=0.04$ ). PsA duration was independently associated with both peripheral and axial joint damage $(\mathrm{p}<0.001)$.

Conclusions: High level of occupation-related mechanical stress is associated with increased radiographic peripheral joint damage. These findings support the potential role of micro-trauma in the pathogenesis of PsA.

Disclosure of Interest: None declared

DOI: 10.1136/annrheumdis-2017-eular.4074

\section{FRI0523 SHORT-TERM EFFICACY AND SAFETY OF NEW BIOLOGICAL AGENTS TARGETING THE IL-6, IL-12/23 AND IL-17 PATHWAYS FOR ACTIVE PSORIATIC ARTHRITIS: A NETWORK META-ANALYSIS OF RANDOMISED CONTROLLED TRIALS}

W. Dongze, Y. Jiang, L.-S. Tam. Department of Medicine \& Therapeutics, The Prince of Wales Hospital, the Chinese University of Hong Kong, Hong Kong, China

Background: IL-6, IL-12/23 and IL-17 inhibitors have been found to be highly effective for both skin and joint manifestations of psoriatic arthritis (PsA). Nonetheless, reliable evidence of the comparative benefits and harms for these interventions is absent for treatment selection in daily practice.

Objectives: To investigate the comparative efficacy, safety and tolerability of IL-6, IL-12/23 or IL-17 inhibitors and the proportion attributable to overall treatment for patients with active PsA.

Methods: Randomized controlled trials (RCTs) evaluating the efficacy, safety and tolerability of IL-6, IL12/23 or IL17 inhibitors at weeks 24 were identified by a comprehensive systematic literature review (PROSPERO 2016: CRD42016048166). Quality of evidence was assessed following the Grading of Recommendations, Assessment, Development and Evaluations (GRADE) approach. Treatment effects were evaluated based on the intention-to treat efficacy rates (ACR20, ACR50), safety parameters (any adverse effect [AE], serious adverse effect [SAE]) and tolerability (discontinuation due to AE [DAE]). Pair-wise meta-analyses and Bayesian network meta-analyses using the random-effects model were performed to estimate pooled odds ratios (ORs) and 95\% credible interval ( $\mathrm{Crl}$ ). A subgroup analysis was performed to investigate the effects of prior anti-TNF exposure on the efficacy of ustekinumab and secukinumab.

Results: Six trials were identified which included 2411 participants and 11 treatments. All trials were of generally high quality according to Cochrane compliant rules and GRADEpro assessment. Direct comparisons of each biologic showed that secukinumab, ustekinumab and ixekizumab demonstrated superior efficacy over placebo with respect to all efficacy outcomes (ACR20 and ACR50). Regarding safety and tolerability, ixekizumab has a higher incidence of adverse events while ustekinumab were more tolerable compared with placebo. Mixed treatment comparisons showed that secukinumab (300mg monthly) had the highest efficacy in achieving ACR20 and ACR50; whereas clazakizumab (200mg monthly), ustekinumab (45mg 12 weekly), secukinumab (150mg monthly) had the lowest probability of having $\mathrm{AE}, \mathrm{SAE}, \mathrm{DAE}$, respectively. Considering the overall risk-benefit profile of IL-6, IL12/23, IL17 inhibitors in the treatment of active PsA, secukinumab (150mg monthly) may offer an optimal balance for active PsA patients (Figure 1). When considering ACR 20 response, subgroup analysis revealed that anti-TNF naïve patient respond significantly better than placebo for all dosages of secukinumab and ustekinumab used. In contrast, only higher dose of secukinumab and ustekinumab were significantly more effective than placebo in anti-TNF failure patient.

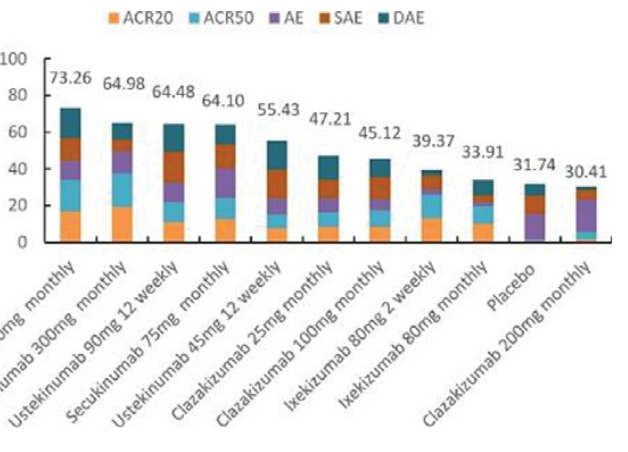

Figure 1

Conclusions: In conclusion, from the available evidence, secukinumab and ustekinumab were found to be the safest and most efficacious short-term treatments for active PsA amongst all the new biologics targeting the IL-6, IL-12.23 and IL-17 pathways.

Acknowledgements: Thank all colleagues working in the Division of Rheuma- tology, Department of Medicine and Therapeutics, The Prince of Wales Hospital, The Chinese University of Hong Kong.

Disclosure of Interest: None declared

DOI: 10.1136/annrheumdis-2017-eular.1469

\section{FRIDAY, 16 JUNE 2017} Osteoporosis

\section{FRI0524 CAROTID ATHEROSCLEROSIS IS ASSOCIATED WITH COMPROMISED VOLUMETRIC BONE MINERAL DENSITY AND MICROSTRUCTURES IN PATIENTS WITH INFLAMMATORY} ARTHRITIS

T.H. Cheng ${ }^{1}$, Q. Shang ${ }^{1}$, E.K.M. Li ${ }^{1}$, P.C.H. Wong ${ }^{1}$, E.W.L. Kun ${ }^{2}$, M. Li ${ }^{1}$, T.K.Y. Li ${ }^{1}$, T.Y. Zhu ${ }^{3}$, A.P.W. Lee ${ }^{1}$, L. Qin ${ }^{3}$, L.S. Tam ${ }^{1} .{ }^{1}$ Department of Medicine and Therapeutics, the Chinese University of Hong Kong; ${ }^{2}$ Department of Medicine \& Geriatrics, Tai Po Hospital; ${ }^{3}$ Department of Orthopaedics and Traumatology, the Chinese University of Hong Kong, Hong Kong, Hong Kong

Background: Carotid Atherosclerosis is associated with compromised volumetric bone mineral density and microstructures in patients with inflammatory arthritis

Objectives: The aim of this study was to explore the relationship between volumetric bone mineral density (VBMD)/microstructural features and presence of carotid plaque $(\mathrm{CP})$ in patients with inflammatory arthritis.

Methods: 175 inflammatory arthritis patients (81 [46\%] PsA, 94 [54\%] RA; 70 [40\%] males; age: $53 \pm 12$ years) were recruited into an ongoing prospective study assessing the relationship between inflammation, osteoporosis and carotid atherosclerosis. Carotid plaque and intima-media thickness (IMT) were measured by carotid ultrasound. Areal BMD (aBMD) was measured by dual energy X-ray absorptiometry (DXA). Microstructure features and vBMD of distal radius were measured using high-resolution peripheral quantitative computed tomography (HR-pQCT).

Results: No patients had established cardiovascular disease (CVD). Data from 172 patients at baseline were analyzed for this cross-sectional study. Patients were sub grouped according to the presence or absence of carotid plaque (CP+ group, $n=68[40 \%]$ ) and CP-group, $n=132$ [60\%]). CP+ group were older ( $59 \pm 10$ vs $49 \pm 11, p<0.001)$, more likely to be male ( $54 \%$ vs $31 \%, p=0.002)$, had higher systolic blood pressure ( $130 \pm 19$ vs $124 \pm 17 \mathrm{mmHg}, p=0.034)$ and CVD risk $(15.7 \pm 14.2$ vs $7.9 \pm 8.6, p<0.001)$ according to the Framingham Risk Score (FRS) then the CP- group. aBMD, vBMD and microstructure were significantly compromised in the $\mathrm{CP}+$ group. Distal radius $\mathrm{aBMD}$, distal radius total VBMD, trabecular (Tb) vBMD, Tb thickness, cortical (Ct.) vBMD, Ct. thickness and bone volume fraction were $5 \%(p=0.004), 12 \%(p<0.001), 8 \%(p=0.007), 8 \%(p=0.004)$, $4 \%(p=0.007), 10 \%(p=0.001)$ and $8 \%(p=0.007)$ lower in the $C P+$ group. The differences remained significant after adjustment for gender, disease type and FRS (Table 1).

Table - Distal radius $\mathrm{VBMD} /$ microstructure and presence of plaque

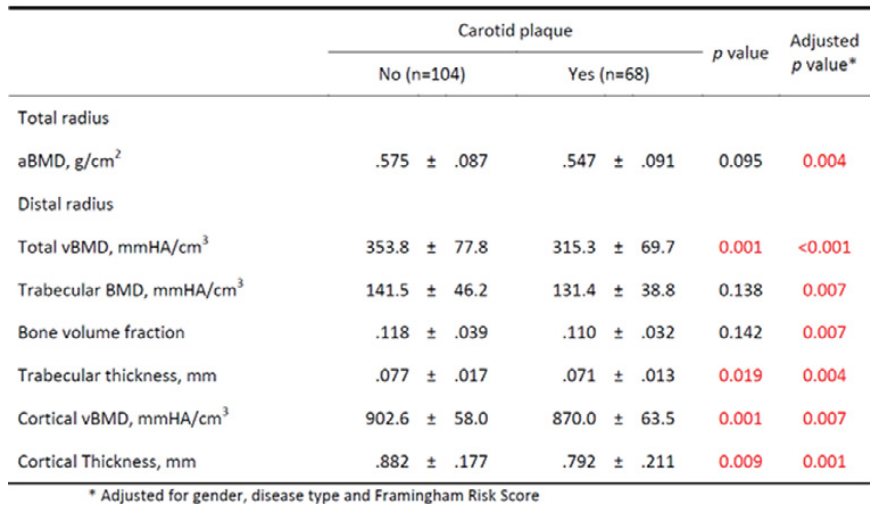

Conclusions: Inflammatory arthritis patients with carotid plaque had lower aBMD, vBMD and compromised bone microstructure in the distal radius even after adjustment for gender, disease type and FRS, suggesting that inflammation may be the common link for both conditions.

Disclosure of Interest: None declared

DOI: 10.1136/annrheumdis-2017-eular.3094 